\title{
Complex Disease
}

National Human Genome Research Institute (NHGRI)

\section{Source}

National Human Genome Research Institute (NHGRI). Complex Disease.

A complex disease is caused by the interaction of multiple genes and environmental factors. Complex diseases are also called multifactorial. Examples of complex diseases include cancer and heart disease. 